relative to wild-type controls. Baseline apoptotic index was also increased 3.4 -fold in the antrum of $\mathrm{p} 50^{-/-}$mice. However, c-Rel ${ }^{-/-}$and $\mathrm{p} 52^{-/-}$mice showed similar baseline gastric mitotic and apoptotic indices to wild-type mice. All mice developed apoptosis in the proliferative zone of antral (cell positions 2-14) and corpus (cell positions 9-24) gastric glands following $\gamma$-radiation and mitosis was completely suppressed in all mice following this stimulus. $6 \mathrm{~h}$ after irradiation, p50/- mice had 1.4-fold higher antral and 4.4-fold higher corpus apoptotic indices (significant interaction between irradiation and genotype by 2 -way ANOVA) than wild-type mice. c-Rel null mice similarly showed 2.2-fold higher corpus apoptosis scores and p52/- mice 2.0-fold higher scores relative to wild-type mice, but these mice showed no differences in radiation-induced antral apoptosis. At $48 \mathrm{~h}$ after irradiation, the only significant differences that persisted were in the corpus of $\mathrm{p} 50^{-/-}$mice.

Conclusion Deletion of NF- $\kappa \mathrm{B} 1, \mathrm{NF}-\kappa \mathrm{B} 2$ and $\mathrm{c}-\mathrm{Rel}$ all render the gastric epithelium more susceptible to radiation-induced apoptosis. Individual family members regulate this process to different extents and the effects are more marked in corpus than antral mucosa. These data suggest that both classical and alternative pathway NF- $\kappa \mathrm{B}$ signalling coordinate the epithelial apoptotic response in the stomach and individual family members play specific roles.

Competing interests None.

Keywords apoptosis, gastric cancer, proliferation.

\title{
OC-017 CLASSICAL AND ALTERNATIVE PATHWAY NF-KB SIGNALLING REGULATE GASTRIC EPITHELIAL RESPONSES TO GAMMA-IRRADIATION
}

doi:10.1136/gut.2011.239301.17

M D Burkitt, ${ }^{1,}$ C A Duckworth, ${ }^{1}$ A Varro, ${ }^{2} \mathrm{~J}$ H Caamano, ${ }^{3}$ D M Pritchard ${ }^{1}$ Department of Gastroenterology, The University of Liverpool, Liverpool, UK; ${ }^{2}$ Department of Cellular and Molecular Physiology, The University of Liverpool, Liverpool, UK; ${ }^{3}$ Unit of Immune Regulation, The University of Birmingham, Birmingham, UK

Introduction Dysregulation of epithelial apoptosis is considered to be an important predisposing event during gastric carcinogenesis. H. pylori infection, the initiating factor for most stomach cancers, induces gastric inflammation and this process is regulated by the NF- $\mathrm{KB}$ family of transcription factors. $H$. pylori also induces gastric epithelial apoptosis and NF- $\kappa$ B family members are key regulators of this cellular process. There are five individual members of the NF- $\kappa B$ family of proteins which signal via either the classical or alternative pathway. We have currently investigated whether individual NF- $\mathrm{\kappa B}$ family members regulate susceptibility towards gastric epithelial apoptosis induced by the standard DNA damaging stimulus of $\gamma$-irradiation.

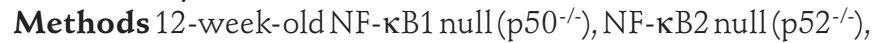
c-Rel null and C57BL/6 mice underwent 12Gy $\gamma$-irradiation and after 6 or $48 \mathrm{~h}$ tissues were harvested and processed for histology. Apoptosis and mitosis were scored on a cell positional basis in the antrum and corpus. Statistical significance was assessed by ANOVA; cell positional data were analysed by modified median test.

Results Epithelial mitotic indices were increased 2.3-fold in the antrum and 3.0 -fold in the corpus of untreated $\mathrm{p} 50^{-/-}$mice 